\title{
HS 0943+1404, a true intermediate polar ${ }^{\star}$
}

\author{
P. Rodríguez-Gil ${ }^{1,2}$, B. T. Gänsicke ${ }^{1}$, H.-J. Hagen ${ }^{3}$, D. Nogami ${ }^{4}$, M. A. P. Torres ${ }^{5}$, \\ H. Lehto ${ }^{6,7}$, A. Aungwerojwit ${ }^{1}$, S. Littlefair ${ }^{8}$, S. Araujo-Betancor ${ }^{9}$, and D. Engels ${ }^{3}$ \\ 1 Department of Physics, University of Warwick, Coventry CV4 7AL, UK \\ e-mail: prguez@iac.es \\ 2 Instituto de Astrofísica de Canarias, Vía Láctea, s/n, La Laguna, 38205 Santa Cruz de Tenerife, Spain \\ ${ }^{3}$ Hamburger Sternwarte, Universität Hamburg, Gojenbergsweg 112, 21029 Hamburg, Germany \\ ${ }^{4}$ Hida Observatory, Kyoto University, Kamitakara, Gifu 506-1314, Japan \\ 5 Harvard-Smithsonian Center for Astrophysics, 60 Garden St., Cambridge, MA 02138, USA \\ 6 Tuorla Observatory, Turku University, Väisäläntie 20, 21500 Piikkiö, Finland \\ 7 Department of Physics, 20014 University of Turku, Finland \\ 8 School of Physics, University of Exeter, Exeter EX4 4QL, UK \\ 9 Space Telescope Science Institute, 3700 San Martin Drive, Baltimore, MD 21218, USA
}

Received 28 March 2005 / Accepted 1 June 2005

\begin{abstract}
We have identified a new intermediate polar, HS 0943+1404, as part of our ongoing search for cataclysmic variables in the Hamburg Quasar Survey. The orbital and white dwarf spin periods determined from time-resolved photometry and spectroscopy are $P_{\text {orb }} \simeq 250 \mathrm{~min}$ and $P_{\text {spin }}=69.171 \pm 0.001 \mathrm{~min}$, respectively. The combination of a large ratio $P_{\text {spin }} / P_{\text {orb }} \simeq 0.3$ and a long orbital period is very unusual compared to the other known intermediate polars. The magnetic moment of the white dwarf is estimated to be $\mu_{1} \sim 10^{34} \mathrm{G} \mathrm{cm}^{3}$, which is in the typical range of polars. Our extensive photometry shows that HS $0943+1404$ enters into deep ( $\sim 3 \mathrm{mag}$ ) low states, which are also a characteristic feature of polars. We therefore suggest that the system is a true "intermediate" polar that will eventually synchronise, that is, a transitional object between intermediate polars and polars. The optical spectrum of HS $0943+1404$ also exhibits a number of unusual emission lines, most noticeably $\mathrm{N}$ II $\lambda 5680$, which is likely to reflect enhanced nitrogen abundances in the envelope of the secondary.
\end{abstract}

Key words. accretion, accretion disc - stars: binaries: close - stars: novae, cataclysmic variables

\section{Introduction}

Our understanding of the evolution of cataclysmic variables (CVs) is still very fragmentary. It is clear that the current theory of CV evolution - largely resting on the disrupted magnetic braking scenario (King 1988) - makes a number of predictions in strong conflict with the observational data. For instance, the theoretical minimum orbital period is $\sim 70 \mathrm{~min}$, much shorter than the observed $\sim 80$ min (Kolb \& Baraffe 1999). In addition, $\mathrm{CV}$ s are expected to spend most of their lifetime near the minimum period, a fact not corroborated by the observations.

* Based in part on observations obtained at the German-Spanish Astronomical Center, Calar Alto, operated by the Max-PlanckInstitut für Astronomie, Heidelberg, jointly with the Spanish National Commission for Astronomy, on observations made with the NOT telescope, operated on the island of La Palma by the Instituto de Astrofísica de Canarias (IAC) at the Spanish Observatorio del Roque de los Muchachos, on observations made with the IAC80 telescope, operated on the island of Tenerife by the IAC at the Spanish Observatorio del Teide, on observations made with the $1.2 \mathrm{~m}$ telescope at the FLWO Observatory, a facility of the Smithsonian Institution.
With regard to the $\mathrm{CV}$ populations above and below the period gap, we observe similar numbers of systems on both sides, while all population syntheses predict that $\sim 95 \%$ of the entire $\mathrm{CV}$ population should have orbital periods below the gap (Kolb 1993; Howell et al. 1997). These three fundamental discrepancies, among others, clearly depict the current misaligning between theory and observations.

Although CV evolution theory has undergone a number of modifications/alternatives (e.g. King \& Schenker 2002; Schenker \& King 2002; Schenker et al. 2002; Andronov et al. 2003), the discrepancies with the properties of the observed $\mathrm{CV}$ population remain unsatisfactorily large, and it seems to be clear that observational selections effects represent a major problem. Gänsicke (2004) has shown that the majority of all CVs have been identified primarily through their variability or through the detection of X-rays, leaving a large parameter space unsampled. We are currently carrying out a large-scale search for CVs based on their spectroscopic properties in the Hamburg Quasar Survey (HQS, Hagen et al. 1995), with the aim of identifying CVs that are inconspicuous in variability or X-ray surveys (Gänsicke et al. 2002b). 
This method proved successful and led to the discovery of a number of systems that show either no or infrequent outbursts, such as e.g. the dwarf nova GY Cnc $=$ HS 0907+1902 (Gänsicke et al. 2000), the SW Sex star HS 0728+6738 (Rodríguez-Gil et al. 2004b), and the enigmatic low-mass transfer system HS 2331+3905 (Araujo-Betancor et al. 2005). Another important class of CVs characterised by rather low levels of variability are those systems containing a magnetic white dwarf, that is rotating either synchronous to the orbital period (polars) or asynchronous (intermediate polars, IPs). The follow-up of HQS CV candidates led to the discovery of a new type of very low mass transfer polars, WX LMi= HS $1023+3900$ (Reimers et al. 1999) and HS 0922+1333 (Reimers \& Hagen 2000), that may be systems shortly before the onset of Roche-lobe overflow from the secondary; the ultra high-field polar RX J1554.2+2721 = HS 1552+2730 (Jiang et al. 2000; Thorstensen \& Fenton 2002; Gänsicke et al. 2004); and the two intermediate polars 1RXS J062518.2+733433= HS 0618+7336 (Araujo-Betancor et al. 2003) and DW Cnc $=$ HS 0756+1624 (Rodríguez-Gil et al. 2004a).

Here we report on the discovery of the third IP, HS 0943+1404, which exhibits very unusual characteristics, and may be one example of the long-sought link between asynchronous and synchronous magnetic CVs.

\section{Observations and data reduction}

\subsection{Photometry}

Differential CCD photometric time series of HS 0943+1404 were obtained during 25 nights throughout the period December 2002 to March 2004, accumulating a total coverage of $\simeq 106 \mathrm{~h}$ (Table 1 ). The photometric observations were carried out at six different telescopes. Brief details about the individual instrumentation and reduction process are given below.

Calar Alto Observatory. At Calar Alto, filterless and $V$-band CCD photometry of HS 0943+1404 was obtained in December 2002 and December 2003 with the CAFOS SITe CCD camera on the $2.2 \mathrm{~m}$ telescope using a small read-out window. The photometric reduction was carried out using the pipeline described by Gänsicke et al. (2004), which, in brief, pre-processes the images in ESO-MIDAS and performs aperture photometry of all stars in the field of view using the Sextractor (Bertin \& Arnouts 1996). Differential magnitudes of HS 0943+1404 were measured relative to the star "C1" (USNO-A2.0 0975$06331245, R=15.9, B=15.9$; see Fig. 1 ), located $\simeq 30$ " southeast of the CV. The comparison star "C1" was checked against the secondary comparison star "C2" (USNO-A2.0 0975$06331238, R=14.3, B=14.6$ ), and no significant variability was detected. In addition, a number of acquisition images of HS 0943+1404 were obtained prior to spectroscopic observations (Sect. 2.2), at both the $2.2 \mathrm{~m}$ and the $3.5 \mathrm{~m}$ telescopes, and the magnitude of the target was determined interactively with the IRAF task imexam, using again the comparison star " $\mathrm{C} 1$ " in Fig. 1.
Table 1. Log of the photometric observations.

\begin{tabular}{|c|c|c|c|c|c|}
\hline UT Date & $\begin{array}{c}\text { Coverage } \\
\text { (h) }\end{array}$ & Filter & $\begin{array}{l}\text { Exp. } \\
\text { (s) }\end{array}$ & \# Frames & $\begin{array}{c}\text { Mean } \\
\text { magnitude }\end{array}$ \\
\hline \multicolumn{6}{|c|}{ Calar Alto $2.2 \mathrm{~m}$} \\
\hline 2001 Apr. 30 & - & $V$ & 30 & 1 & 17.0 \\
\hline 2002 Dec. 14 & - & $V$ & 30 & 1 & 17.6 \\
\hline 2002 Dec. 15 & - & $V$ & 30 & 1 & 17.6 \\
\hline 2002 Dec. 28 & 1.16 & $V$ & 45 & 64 & 19.2 \\
\hline 2003 Mar. 06 & - & Clear & 20 & 1 & 19.1 \\
\hline 2003 Dec. 17 & 1.11 & Clear & 30 & 88 & 16.1 \\
\hline 2003 Dec. 23 & - & Clear & 30 & 2 & 16.6 \\
\hline 2003 Dec. 23 & - & $V$ & 30 & 2 & 16.5 \\
\hline \multicolumn{6}{|c|}{ Calar Alto $3.5 \mathrm{~m}$} \\
\hline 2004 Mar. 02 & - & $V$ & 30 & 1 & 16.7 \\
\hline 2004 Mar. 03 & - & $V$ & 30 & 1 & 16.5 \\
\hline \multicolumn{6}{|c|}{ FLWO $1.2 \mathrm{~m}$, CCD } \\
\hline 2004 Mar. 09 & 4.96 & Clear & 60 & 407 & 16.2 \\
\hline 2004 Mar. 10 & 7.70 & Clear & 60 & 471 & 16.3 \\
\hline 2004 Mar. 19 & 3.72 & Clear & 60 & 270 & 16.4 \\
\hline 2004 Mar. 20 & 7.08 & Clear & 60 & 605 & 16.3 \\
\hline \multicolumn{6}{|c|}{ IAC80 $0.8 \mathrm{~m}, \mathrm{CCD}$} \\
\hline 2003 Dec. 27 & 5.81 & Clear & 40 & 500 & 16.3 \\
\hline 2003 Dec. 28 & 3.19 & Clear & 40 & 261 & 16.4 \\
\hline \multicolumn{6}{|c|}{ Tuorla $0.7 \mathrm{~m}, \mathrm{CCD}$} \\
\hline 2004 Jan. 20 & 3.97 & Clear & 60 & 217 & 16.5 \\
\hline 2004 Feb. 09 & 6.89 & Clear & 60 & 360 & 16.4 \\
\hline 2004 Mar. 16 & 5.76 & Clear & 60 & 299 & 16.5 \\
\hline 2004 Mar. 18 & 7.83 & Clear & 60 & 395 & 16.5 \\
\hline \multicolumn{6}{|c|}{ Hida 0.6 m, CCD } \\
\hline 2004 Mar. 09 & 6.64 & Clear & 60 & 321 & 16.4 \\
\hline 2004 Mar. 13 & 2.26 & Clear & 60 & 113 & 16.3 \\
\hline 2004 Mar. 15 & 3.67 & Clear & 60 & 191 & 16.4 \\
\hline 2004 Mar. 19 & 2.67 & Clear & 60 & 136 & 16.4 \\
\hline 2004 Mar. 26 & 0.52 & Clear & 60 & 24 & 16.5 \\
\hline 2004 Mar. 27 & 6.17 & Clear & 60 & 296 & 16.3 \\
\hline 2004 Mar. 28 & 6.60 & Clear & 60 & 306 & 16.4 \\
\hline \multicolumn{6}{|l|}{ JKT $1.0 \mathrm{~m}$} \\
\hline 2002 Dec. 29 & 2.72 & Clear & 60 & 120 & 19.0 \\
\hline 2002 Dec. 31 & 6.25 & Clear & 60 & 296 & 19.0 \\
\hline 2003 Jan. 04 & 2.75 & Clear & 45 & 148 & 18.2 \\
\hline 2003 Jan. 05 & 0.40 & Clear & 30 & 32 & 18.7 \\
\hline 2003 Jan. 07 & 3.52 & Clear & 45 & 205 & 18.9 \\
\hline 2003 Jan. 09 & 2.76 & Clear & 60 & 91 & 19.1 \\
\hline
\end{tabular}

Observatorio del Roque de los Muchachos. On La Palma, filterless CCD photometry of HS $0943+1404$ was obtained in December 2002/January 2003 using the Jacobus Kapteyn Telescope (JKT) with the $2 \mathrm{k} \times 2 \mathrm{k}$ pixel $^{2}$ SITe detector. Observations and data reduction were carried out in an analogous fashion as described above for the Calar Alto photometry. 


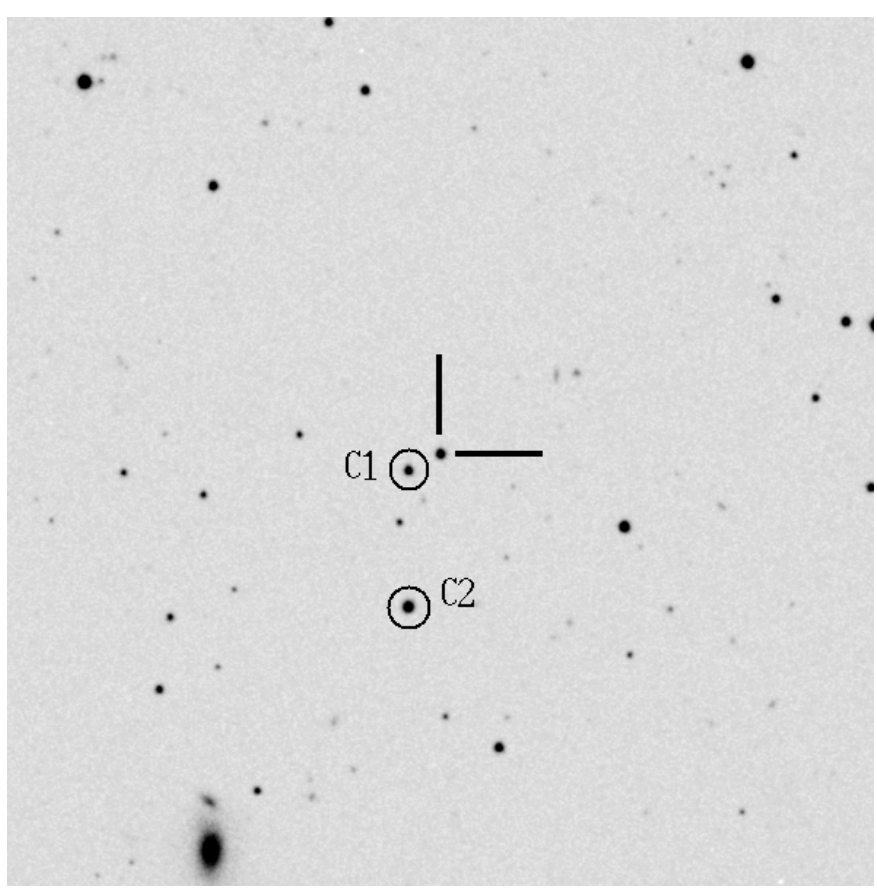

Fig. 1. $10^{\prime} \times 10^{\prime}$ finding chart of HS $0943+1404$ obtained from the Digitized Sky Survey. The coordinates of the CV are $\alpha(\mathbf{J} 2000)=$ $09^{\mathrm{h}} 46^{\mathrm{m}} 34.5^{\mathrm{s}}, \delta(\mathrm{J} 2000)=+13^{\circ} 50^{\prime} 58.1^{\prime \prime}$. North is up and east to the left. " $\mathrm{C} 1$ " is the primary comparison star and " $\mathrm{C} 2$ " is the check star.

Fred Lawrence Whipple Observatory. Filterless observations of HS $0943+1404$ were carried out with the $1.2 \mathrm{~m}$ telescope at the Fred Lawrence Whipple Observatory (FLWO) in March 2004. The 4-Shooter CCD camera was in place, which consists of an array of four $2048 \times 2048$ pixel $^{2}$ CCDs. Only a small window of the CCD \#3 was read out. Data reduction was carried out using IRAF. After bias and flat-field corrections, the images were aligned and instrumental magnitudes of HS 0943+1404 and the comparison stars " $\mathrm{C} 1$ " and "C2" were extracted using the point spread function (PSF) packages. Differential magnitudes of HS $0943+1404$ were then computed relative to "C1".

Observatorio del Teide. Filterless CCD photometry of HS 0943+1404 was obtained at the $0.8 \mathrm{~m}$ IAC80 telescope at the Observatorio del Teide on Tenerife in December 2003, us-

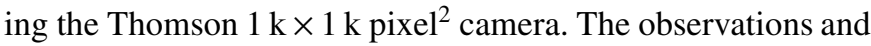
data reduction were carried out in an analogous way as described above for the FLWO photometry.

Tuorla Observatory. The $0.7 \mathrm{~m}$ Schmidt-Vaisala telescope at Tuorla Observatory was used in January, February and March 2004 to obtain filterless photometry of HS 0943+1404 using a SBIG ST-8 CCD camera. Observations and data reduction were carried out in the same way as described above for the Calar Alto photometry.

Hida Observatory. We performed filterless CCD photometry of HS $0943+1404$ with the $0.6 \mathrm{~m}$ telescope and the SITe CCD camera at Hida Observatory (Japan) in March 2004. After standard reduction of the raw images, differential aperture
Table 2. $\log$ of the spectroscopic observations.

\begin{tabular}{lcccc}
\hline \hline UT Date & $\begin{array}{c}\text { Coverage } \\
(\mathrm{h})\end{array}$ & Grating & $\begin{array}{c}\text { Exp. } \\
(\mathrm{s})\end{array}$ & \# Frames \\
\hline \multicolumn{7}{c}{ Calar Alto 2.2 m, CAFOS } \\
2001 Apr. 30 & - & B-200 & 600 & 1 \\
2002 Dec. 14 & 0.75 & G-100 & 600 & 5 \\
2002 Dec. 15 & 0.76 & G-100 & 600 & 5 \\
2003 Dec. 23 & 4.67 & G-100 & 600 & 22 \\
NOT 2.5 m, ALFOSC & & & \\
2003 Dec. 16 & 4.12 & Grism \#7 & 600 & 24 \\
2003 Dec. 17 & 6.03 & Grism \#7 & 300 & 64 \\
Calar Alto 3.5 m, MOSCA & & \\
2004 Mar. 01 & 0.37 & G-1000 & 300 & 4 \\
2004 Mar. 02 & 2.08 & G-1000 & 450 & 15 \\
2004 Mar. 03 & 3.18 & G-1000 & 450 & 14 \\
\hline
\end{tabular}

photometry was carried out relative to the comparison star "C1" in a standard manner using IRAF.

\subsection{Optical spectroscopy}

Calar Alto Observatory. An identification spectrum of HS 0943+1404 was obtained on 2001 April 30 at the $2.2 \mathrm{~m}$ telescope using the Calar Alto Faint Object Spectrograph (CAFOS) equipped with the standard SITe $2 \mathrm{k} \times 2 \mathrm{k}$ pixel $^{2} \mathrm{CCD}$ (Table 2). The B-200 grating in conjunction with a $1.5^{\prime \prime}$ slit provided a spectral resolution of $\simeq 10 \AA$ (full width at half maximum, $F W H M$ ) over the wavelength range $\lambda \lambda 3800-7000$. The spectrum was reduced online using the MIDAS quicklook context available at the telescope. The detection of strong Balmer and helium emission lines revealed the likely $\mathrm{CV}$ nature of HS $0943+1404$.

CAFOS on the $2.2 \mathrm{~m}$ telescope was again used in December 2002 and December 2003 to obtain time-resolved, followup spectroscopy. The G-100 grism and a slit width of 1.2 " covered the wavelength range $\lambda \lambda 4300-8300$ at a spectral resolution of $\simeq 4.5 \AA F W H M$. The raw images were biassubtracted and flat-field compensated before removing the sky contribution. The spectra of HS 0943+1404 were optimally extracted according to the algorithm explained in Horne (1986). To achieve a proper wavelength calibration, a low-order polynomial was fitted to the arc data (the rms being less than one tenth of the dispersion in all cases). The pixel-wavelength dependence for each target spectrum was obtained by interpolating between the two nearest arc spectra. The reduction and extraction were performed within $\operatorname{IRAF}^{1}$ and the wavelength calibration was done in MOLLY.

Additional time-resolved spectroscopy was carried out in March 2004 using MOSCA on the $3.5 \mathrm{~m}$ telescope. The data were obtained with the $2 \mathrm{k} \times 4 \mathrm{k}_{\text {pixel }^{2}}$ SITe CCD camera. The slit width was fixed to $1^{\prime \prime}$ and the G-1000 grism was in place.

1 IRAF is distributed by the National Optical Astronomy Observatories. 
This setup gave a wavelength range of $\lambda \lambda 4200-7400$ at a spectral resolution of $\simeq 2.6 \AA$ (FWHM). The data reduction was carried out in the same way as described for the time-resolved CAFOS spectroscopy.

Observatorio del Roque de los Muchachos. The Andalucía Faint Object Spectrograph and Camera (ALFOSC) along with the $2048 \times 2048$ pixel $^{2}$ EEV chip $(\mathrm{CCD} \# 8)$ were used at the Nordic Optical Telescope (NOT) on La Palma to obtain timeresolved spectroscopy of HS 0943+1404 in December 2003. The combination of grism \#7 (plus the second-order blocking filter WG345) and a $1^{\prime \prime}$ slit provided a resolution of $\simeq 3.7 \AA$ (FHWM) and a useful wavelength range of $\lambda \lambda 3800-6800$. As the arc lines projected onto more than 4 pixels $(F W H M)$, a $1 \times 2$ binning (dispersion direction) was applied. This provided a better signal-to-noise ratio without significantly degrading the spectral resolution. The data reduction was carried out in the same way as explained above.

\section{Analysis: photometry}

\subsection{Long-term variability}

From the nightly mean magnitudes presented in Table 1 it is apparent that HS $0943+1404$ exhibits pronounced long-term changes in its average brightness. The system was observed at an intermediate magnitude of $V \simeq 17.6$ in mid-December 2002, and in a faint state at $V \simeq 19.0$ from late-December 2002 until March 2003. The transition from the intermediate to the low state hence occured in less than two weeks. By the time of our next observations in December 2003, the system was back to a bright state near $V \simeq 16.3$, where it apparently remained until our last observations in March 2004. Similar long-term variability is observed in strongly magnetic, disc-less CVs (polars) and VY Scl stars (see e.g. Warner 1999). We will hereafter follow the common terminology for these objects, calling the bright state high state and the faint state low state.

\subsection{Short-term variability}

In addition to the occurence of high and low states, HS 0943+1404 displays a complex range of short-term variations which we have analysed in detail by computing Scargle periodograms (Scargle 1982) using Schwarzenberg-Czerny's (1989) implementation in the MIDAS context TSA. After an initial assessment of the periodograms, we have split the photometric data (Table 1) into three separate subsets according to the average brightness of the system, which we discuss below.

\subsubsection{December 2002/January 2003 - the low state}

The JKT light curves of HS 0943+1404 obtained during the low state show short-term variability superimposed on a low-amplitude modulation (Fig. 2). The Scargle periodogram (Fig. 3, top panel) shows power in three clusters near $\sim 5 \mathrm{~d}^{-1}$, $\sim 15 \mathrm{~d}^{-1}$, and $\sim 22 \mathrm{~d}^{-1}$. The shortness of the individual low-state observations and their poor sampling result in a substantial

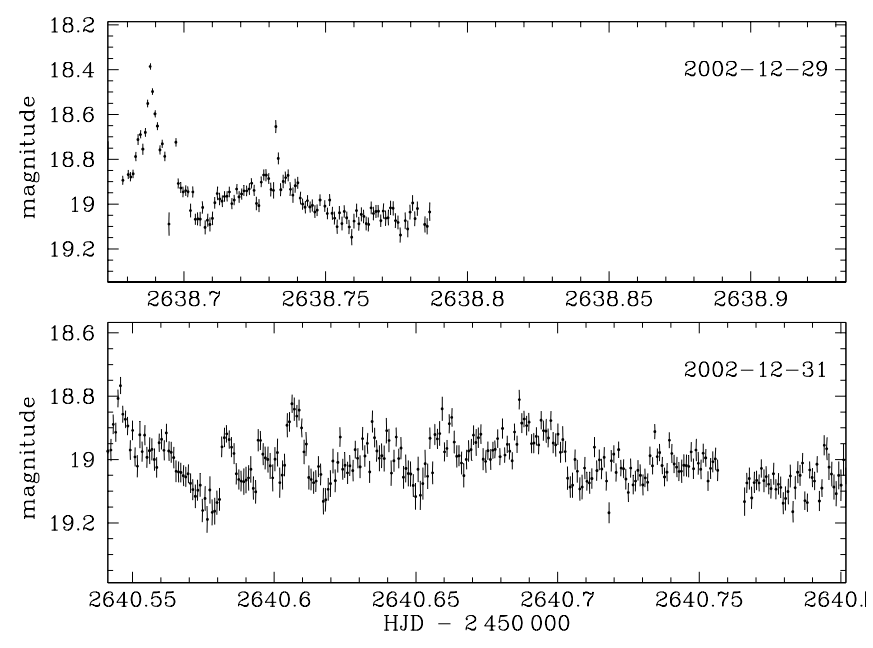

Fig. 2. The low-state photometry obtained with the JKT in December 2002/January 2003 shows evidence of short-lived flares (top), and a low-amplitude, long-period (several hours) modulation superimposed on short-period variability.

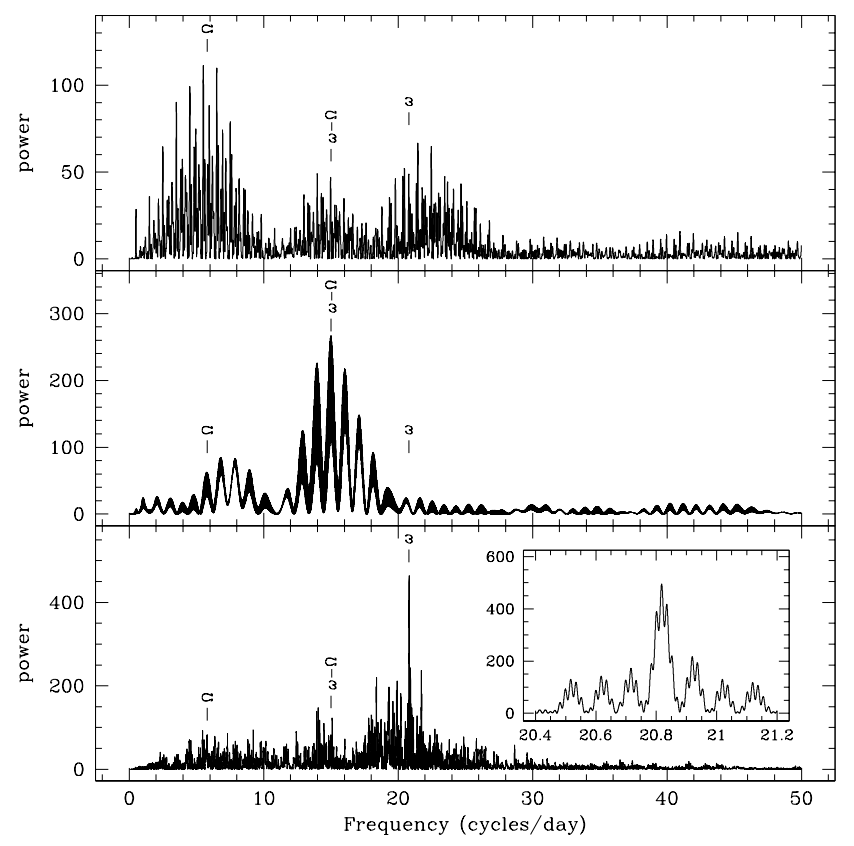

Fig. 3. Scargle periodograms computed from three subsets of the CCD photometry listed in Table 1. Top panel: periodogram for the low state data obtained in December 2002/January 2003. Three clusters of signals are evident at $\sim 5 \mathrm{~d}^{-1}, \sim 15 \mathrm{~d}^{-1}$, and $\sim 22 \mathrm{~d}^{-1}$. Middle panel: periodogram of the December 2003 IAC80 data and the February 2004 Tuorla data. The dominant signal is found at $15 \mathrm{~d}^{-1}$ (plus one-day aliases), and a weaker signal at $6.8 \mathrm{~d}^{-1}$ (plus one-day aliases). Bottom panel: periodogram for the January 2004 Tuorla data and the March 2004 campaign. The strongest signal is detected at $20.8 \mathrm{~d}^{-1}$, a second cluster of signals is present near $15 \mathrm{~d}^{-1}$. The inset shows a close-up of the $20.8 \mathrm{~d}^{-1}$ signal. We identify the $20.8 \mathrm{~d}^{-1}$ frequency with the white dwarf spin $\omega\left(P_{\text {spin }}=69.2 \mathrm{~min}\right)$, and the $15 \mathrm{~d}^{-1}$ frequency with the beat between the white dwarf spin and the orbital motion $\omega-\Omega$, which implies an orbital frequency of $\Omega=5.8 \mathrm{~d}^{-1}\left(P_{\text {orb }}=248 \mathrm{~min}\right)$.

number of aliases in all three ranges, impeding a more detailed analysis. 


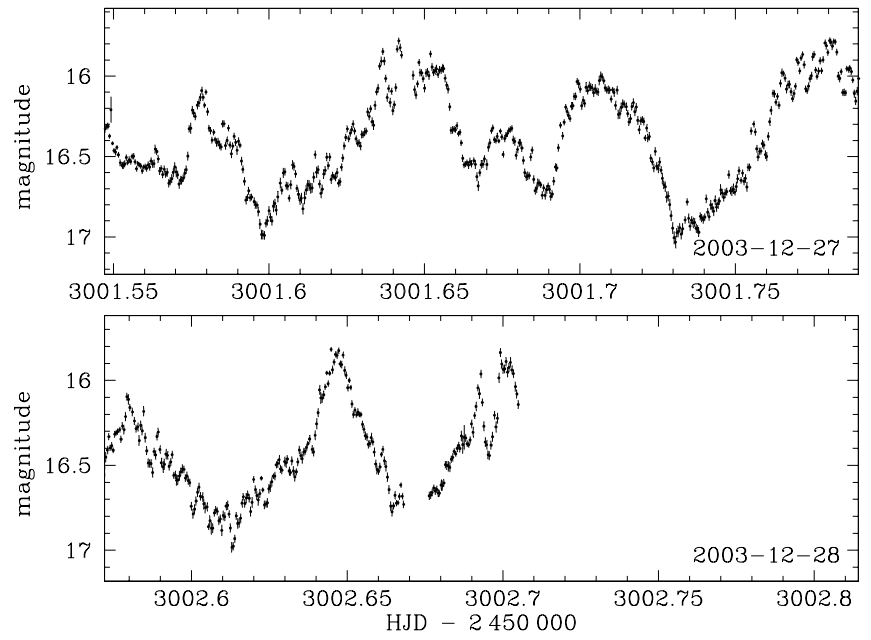

Fig. 4. During the two nights of IAC80 observations, HS 0943+1404 displayed a photometric variability with a period of $\simeq 96 \mathrm{~min}$, which we interpret as the beat period.

\subsubsection{December 2003 \& February 2004 - a 96 min modulation in high state}

The light curves obtained during the December 2003 IAC80 (Fig. 4) and the February 2004 Tuorla runs display a largeamplitude $(\simeq 0.5 \mathrm{mag})$ modulation with a period of $\simeq 96 \mathrm{~min}$. The Scargle periodogram of these three nights of data (Fig. 3, middle panel) confirms this visual estimate, being dominated by a signal at $15 \mathrm{~d}^{-1}$ (plus one-day aliases thereof). A second weaker signal is found at $6.8 \mathrm{~d}^{-1}$, also flanked by one-day aliases. Variability on shorter time scales than the dominant $96-$ min modulation is seen in the light curves, but the periodogram contains no strong signal at higher frequencies.

\subsubsection{January \& March 2004 campaign - a 69 min modulation in high state}

The light curves obtained in January 2004 at the Tuorla Observatory and during the March 2004 multi-site campaign carried out at FLWO, Hida Observatory, and Tuorla Observatory are dominated by a coherent signal with a period of $\simeq 69$ min and an amplitude of $0.2-0.3 \mathrm{mag}$ (Fig. 5). The Scargle periodogram of this data set (Fig. 3 , bottom panel) is dominated by a sharp signal at $20.8 \mathrm{~d}^{-1}$, and contains some weaker signals in the range $14-15.2 \mathrm{~d}^{-1}$.

\subsection{An intermediate polar interpretation}

Complex short-term variability characterized by the presence of several coherent signals is the hallmark of IPs, CVs containing a moderately magnetic white dwarf with a spin period shorter than the orbital period, $P_{\text {spin }}<P_{\text {orb }}$. The power spectra of IPs typically show signals at the orbital frequency $\Omega$, the white dwarf spin frequency $\omega$, and the beat (i.e. synodic) frequency $\omega-\Omega$ (additional beat signals at $\omega+\Omega$ or $\omega-2 \Omega$ have been detected in some IPs, e.g. Warner 1986).

Considering the morphology of the observed light curves and the resulting periodograms, we suggest that HS 0943+1404
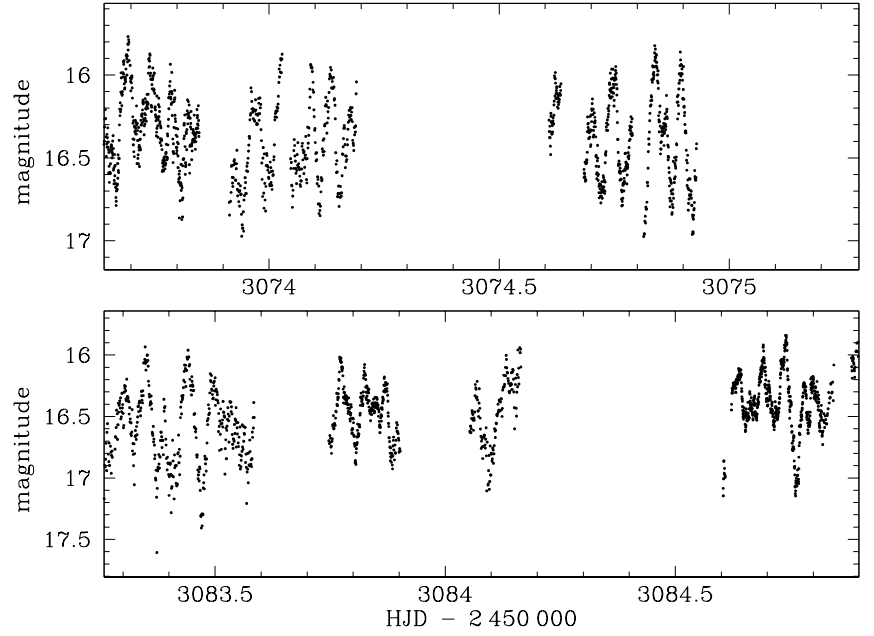

Fig. 5. Sample light curves obtained at FWLO, Hida Observatory, and Tuorla observatory during the March 2004 campaign. The data show a periodic modulation with a period of $\simeq 69 \mathrm{~min}$, which we identify with the white dwarf spin period.

is an IP. We identify the highly coherent, highest frequency signal detected in the photometry of HS $0943+1404$ with the white dwarf spin, the intermediate frequency signal as the beat between white dwarf spin and orbital periods, and the lowest frequency signal with the orbital period. The spin signal in the bottom panel of Fig. 3 shows some alias substructure due to the long gap between the January 2004 and March 2004 data. The frequency of the central peak is $20.8179 \pm 0.0003 \mathrm{~d}^{-1}$, with the two flanking aliases located at $20.8011 \pm 0.0003 \mathrm{~d}^{-1}$ and $20.8348 \pm 0.0003 \mathrm{~d}^{-1}$, where the errors given in brackets have been determined by fitting a sine wave to the data. The beat signal (Fig. 3, middle panel) is also plagued by multiple aliases due to the separation of the December 2003 and February 2004 runs. The strongest signal is found at $15.0 \pm 0.2 \mathrm{~d}^{-1}$, where the error in brackets is conservatively estimated from half the FWHM of the central cluster of aliases.

We conclude that HS $0943+1404$ is an IP, and the analysis of our photometric data results in $P_{\text {spin }}=69.171 \pm 0.001 \mathrm{~min}$, $P_{\text {beat }}=96.0 \pm 3.3 \mathrm{~min}$, and $P_{\text {orb }}=247.5 \pm 3.3 \mathrm{~min}$. Figure 6 shows the spin and beat-dominated data folded on the spin and beat period, respectively.

\section{Analysis: spectroscopy}

\subsection{The optical spectrum of HS $0943+1404$}

The spectrum of HS $0943+1404$ (Fig. 7) is dominated by strong, single-peaked emission lines of H I and He I. He II line emission at $\lambda 4686,5412$, and the Bowen blend are also prominent, indicating the presence of a source of high-energy photons. The relative strength of these emission lines is reminiscent of the magnetic CVs. The continuum significantly rises bluewards of $\mathrm{H} \alpha$ and atmospheric features of the secondary star are absent in the observed spectral ranges.

The most remarkable feature in the optical spectrum of HS 0943+1404 is the strength of several emission lines that are either very weak or absent in most other CVs, such as Fe II 

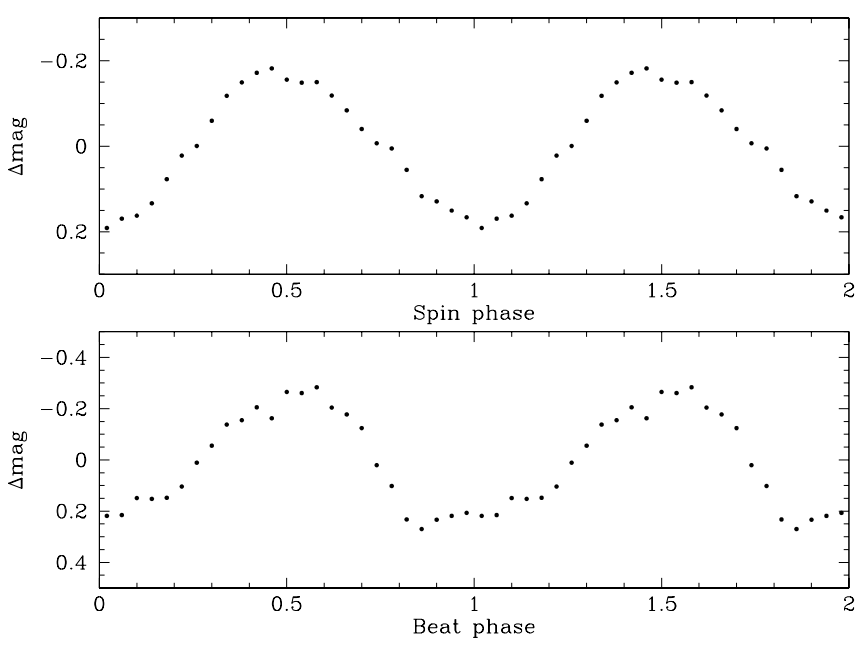

Fig. 6. Top panel: the January 2004 and March 2004 data folded on the white dwarf spin period of $69.2 \mathrm{~min}$. Bottom panel: the December 2003 and February 2004 data folded on the beat period of $96 \mathrm{~min}$.

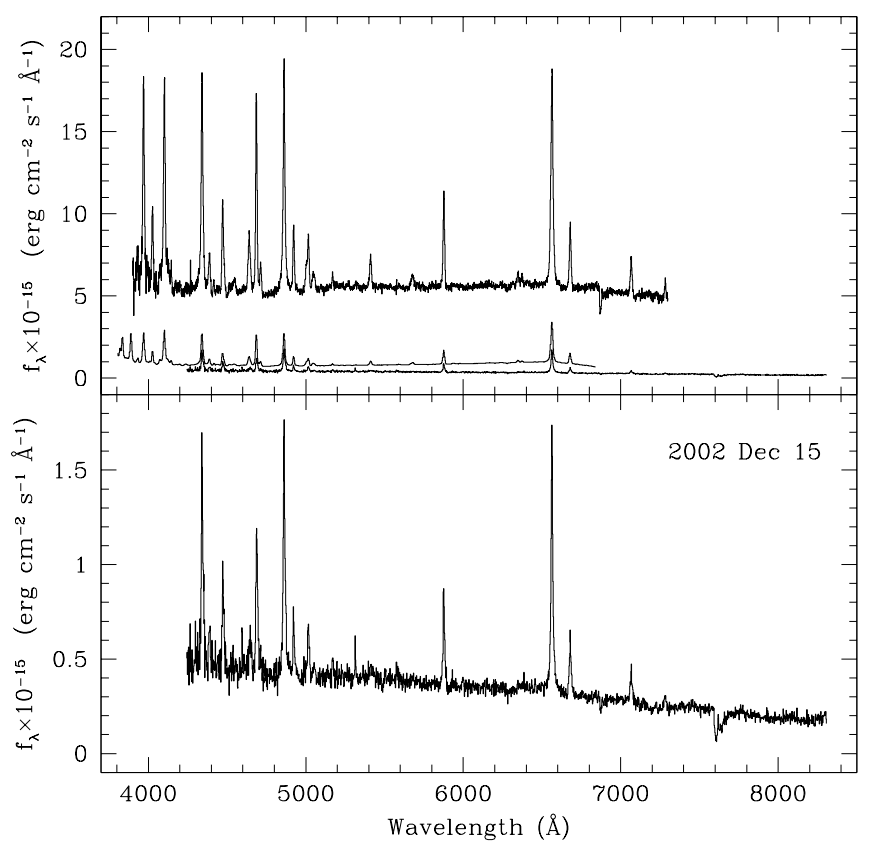

Fig. 7. Top panel: flux calibrated average spectra of HS $0943+1404$ taken in different epochs. From bottom to top, 2002 December 15 (Calar Alto 2.2 m), 2003 December 16-17 (NOT), and 2004 March 1-3 (Calar Alto $3.5 \mathrm{~m}$ ). These spectra nicely illustrate the rather frequent brightness changes of HS 0943+0414. Bottom panel: enlarged version of the lower spectrum shown in the top panel (2002 December). The system was in an intermediate state near $V=$ 17.6 mag. No spectroscopic signature of the donor star is evident in the red part of the spectrum.

(e.g. $\lambda 5018,5169), \mathrm{N}_{\text {II }} \lambda$ 15680, Si II $\lambda 6347,6372$, and probably Al II $\lambda 6243$ (see the $\lambda \lambda 4900-6500$ range of the 2003 December NOT average spectrum; Fig. 8). A closer inspection reveals that the spectrum of HS 0943+1404 shows abundant weak emission lines throughout. In Table 3 we present the equivalent widths $(E W s)$ of the most prominent lines as measured in the average spectra corresponding to the three different epochs.

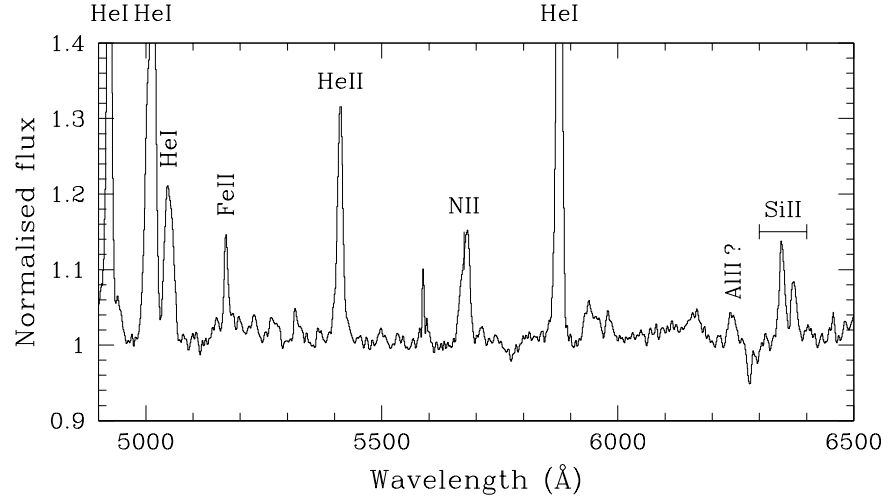

Fig. 8. Average spectrum of HS 0943+1404 (2003 December, NOT) showing a number of unusual emission lines in the range $\lambda \lambda 4900-6500$. Note the strong N II $\lambda 5680$ and Si II $\lambda 6347$ and $\lambda 6371$.

Table 3. Line equivalent widths measured from the average spectra.

\begin{tabular}{lccr}
\hline \hline Line & $\begin{array}{c}E W \\
(\AA)\end{array}$ & Line & $\begin{array}{r}E W \\
(\AA)\end{array}$ \\
\hline $\mathbf{2 0 0 2}$ December (intermediate & state) & \\
H $\alpha$ & 70 & He I $\lambda 6678$ & 13 \\
$\mathrm{H} \beta$ & 48 & He I $\lambda 5876$ & 18 \\
H $\gamma$ & 33 & He I $\lambda 4922$ & 7 \\
He II $\lambda 4686$ & 25 & He I $\lambda 4472$ & 11 \\
He II $\lambda 5412$ & 4 & He I $\lambda 4388$ & 3 \\
Bowen blend & 6 & N II $\lambda 5680$ & 2 \\
He I $\lambda 7065$ & 10 & & \\
$\mathbf{2 0 0 3}$ December $($ high state) & & \\
H $\alpha$ & 53 & He I $\lambda 7281$ & 5 \\
H $\beta$ & 49 & He I $\lambda 7065$ & 9 \\
H $\gamma$ & 44 & He I $\lambda 6678$ & 12 \\
H $\delta$ & 42 & He I $\lambda 5876$ & 16 \\
H $\varepsilon$ & 32 & He I $\lambda 4922$ & 10 \\
H8 & 22 & He I $\lambda 4472$ & 16 \\
H9 & 13 & He I $\lambda 4388$ & 7 \\
He II $\lambda 4686$ & 35 & He I $\lambda 4026$ & 11 \\
He II $\lambda 5412$ & 6 & N II $\lambda 5680$ & 4 \\
Bowen blend & 16 & & \\
$\mathbf{2 0 0 4}$ March (high state) & & & \\
H $\alpha$ & 52 & He I $\lambda 6678$ & 13 \\
H $\beta$ & 51 & He I $\lambda 5876$ & 14 \\
He II $\lambda 4686$ & 31 & He I $\lambda 4922$ & 10 \\
He II $\lambda 5412$ & 8 & He I $\lambda 4472$ & 19 \\
Bowen blend & 17 & He I $\lambda 4388$ & 9 \\
He I $\lambda 7065$ & 9 & N II $\lambda 5680$ & 5 \\
\hline
\end{tabular}

\subsection{Radial velocity variations}

In order to search for orbital variability, we measured the radial velocities of the $\mathrm{H} \alpha, \mathrm{H} \beta, \mathrm{He}$ I $\lambda 5876$ and $\mathrm{He}$ II $\lambda 4686$ emission lines. The spectra were first re-binned to a constant velocity scale centred at the rest wavelength of each line and normalised. Radial velocities were measured by using three different methods: (i) correlation with a single Gaussian template; (ii) the double Gaussian technique of Schneider \& Young (1980); and (iii) calculation of the median velocity (the point leaving the same area on both sides of the normalised line 


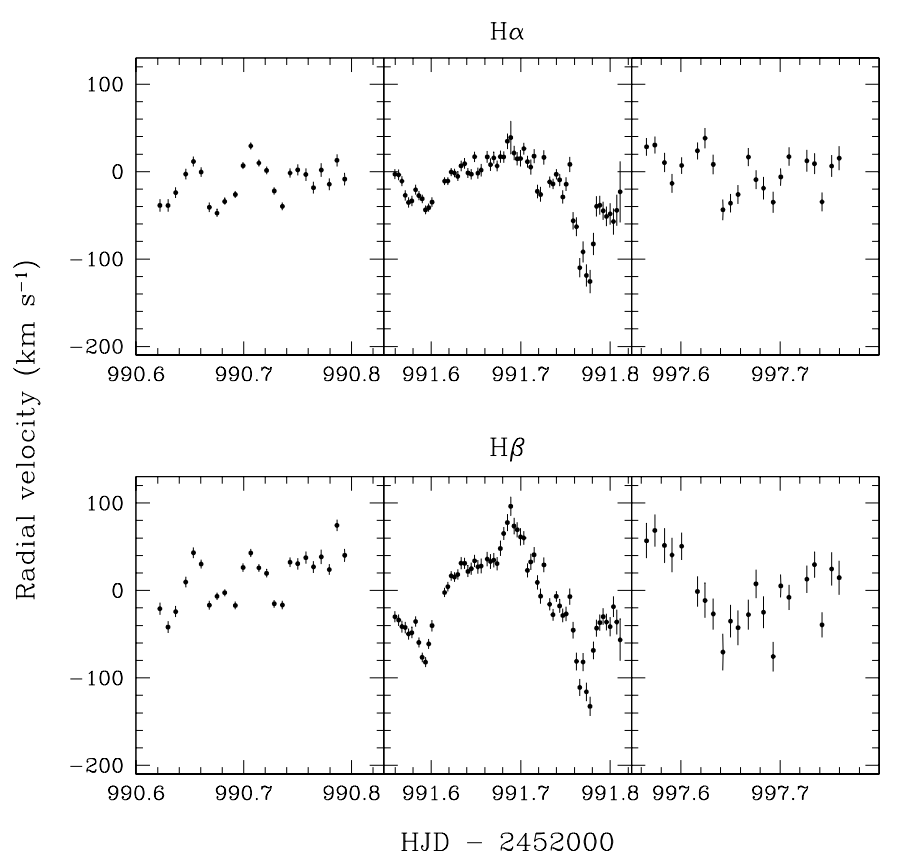

Fig. 9. $\mathrm{H} \alpha$ (top) and $\mathrm{H} \beta$ (bottom) radial velocity curves. From left to right: 2003 December 16, 17, and 23.

profile). The latter method proved to be the most efficient, producing the cleanest radial velocity curves. The resulting curves for $\mathrm{H} \alpha$ and $\mathrm{H} \beta$ are shown in Fig. 9.

The morphology of the radial velocity curves seems to significantly change from night to night. On 2003 December 16, a modulation at $\sim 70$ min dominates, whereas a longer time scale modulation is clearly seen on 2003 December 17. On this night, a dip towards blue velocities at HJD $\simeq 2452991.77$ is observed. A shallower excursion to the blue also occurs at $\mathrm{HJD} \simeq 2452991.59$. The separation between them was measured by fitting Gaussians to the dips, obtaining a value of $\simeq 0.19 \mathrm{~d}$. In addition, the $\mathrm{H} \beta$ curve displays a red spike centred at $\simeq 2452991.69$, almost just in the middle of the blue dips. A sine fit to the $\mathrm{H} \beta$ radial velocity curve (after masking the dips) yields a period of $\simeq 0.19 \mathrm{~d}$ and a semi-amplitude of $\sim 45 \mathrm{~km} \mathrm{~s}^{-1}$.

In order to search for periodicities we computed Scargle periodograms (Scargle 1982) for all the $\mathrm{H} \alpha, \mathrm{H} \beta, \mathrm{He}$ I $\lambda 5876$, and He II $\lambda 4686$ radial velocity curves. The periodograms for all the lines are very similar, and we present in Fig. 10 only the periodograms for $\mathrm{H} \beta$ and $\mathrm{He}$ II $\lambda 4686$. Both periodograms are dominated by clusters of aliases near $\simeq 3.4,4.4$, and $5.4 \mathrm{~d}^{-1}$. The poor sampling of our radial velocities spread out over 15 months causes severe fine-structure in the main alias clusters, and impedes an unequivocal identification of the frequency inherent to the system. Formally, the strongest signal in the $\mathrm{H} \beta$ radial velocity variation is found at $5.430 \pm 0.002 \mathrm{~d}^{-1}$ (the quoted error is a conservative estimate from the width of the peak in the periodogram), which corresponds to a period of $P=0.18416 \pm 0.00007 \mathrm{~d}(=265.2 \pm 0.1 \mathrm{~min})$. In the case of He II $\lambda 4686$, a value of $4.412 \pm 0.005 \mathrm{~d}^{-1}(P=$ $0.2267 \pm 0.0003 \mathrm{~d}=325.8 \pm 0.4 \mathrm{~min}$ ) is found. The presence of power around $250 \mathrm{~min}$ in both the low-state photometry (Fig. 3, top panel) and in the radial velocity variations (Fig. 10) strongly suggests the detection of a "clock" in HS 0943+1404.
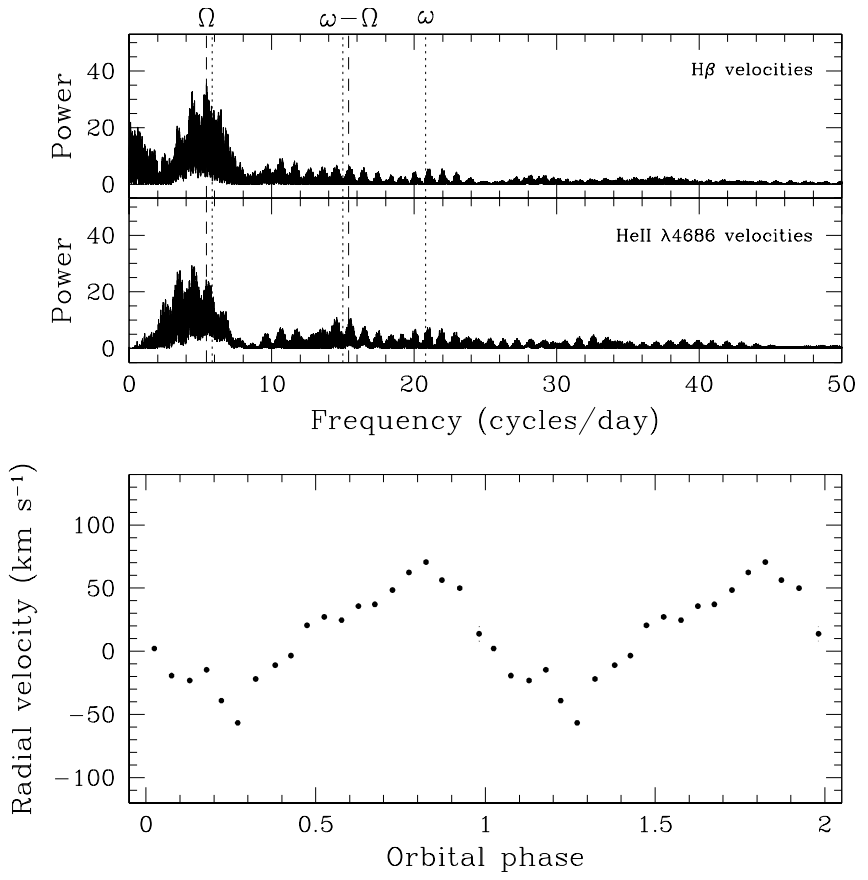

Fig. 10. Top: scargle periodograms of the $\mathrm{H} \beta$ and He II $\lambda 4686$ radial velocity curves. $\Omega$ is the orbital frequency, $\omega$ the white dwarf spin, and $\omega-\Omega$ the corresponding synodic frequency. Dashed (dotted) vertical lines correspond to spectroscopic (photometric) results. Bottom: $\mathrm{H} \beta$ radial velocity curve folded on 265.2 min after averaging the data into 20 phase bins. The time of red-to-blue crossing is $T_{0}=2452623.78160$ (HJD). The orbital cycle has been plotted twice for continuity.

Given that this is the longest period detected in all our data we believe that it represents the orbital motion of the binary.

The $\mathrm{H} \beta$ radial velocities folded on $265.2 \mathrm{~min}$, the strongest signal in the $\mathrm{H} \beta$ periodogram, displays a relatively lowamplitude modulation superimposed by a short $(\sim 0.15$ orbital phase) negative excursion, which is mostly related to the two "dips" seen in the December 17 NOT data.

\section{3. $V / R$ ratios and equivalent width curves}

Our second approach at searching for periodicities involved the analysis of the $\mathrm{H} \alpha, \mathrm{H} \beta, \mathrm{He}$ I $\lambda 5876$ and $\mathrm{He}$ II $\lambda 4686 V / R$-ratio and $E W$ curves. The $V / R$ ratio is computed by dividing the $E W$ of the blue half of the line profile (up to zero velocity, i.e. the $V$ part) by that of the red half (redwards from zero velocity; the $R$ part). Only the Scargle periodograms for $\mathrm{H} \beta$ and $\mathrm{He}$ II $\lambda 4686$ are shown in Fig. 11. The picture in the low frequency regime does not shed new light on the orbital period discussion, as it contains the same three clusters of aliases around 3.4, 4.4, and $5.4 \mathrm{~d}^{-1}$. However, the $E W$ periodograms contain a strong peak at the photometric spin frequency. The strongest peak in both the $\mathrm{H} \beta$ and $\mathrm{He}$ II $\lambda 4686 \mathrm{EW}$ periodograms is centred at $20.82 \mathrm{~d}^{-1}$, exactly at the spin frequency. 


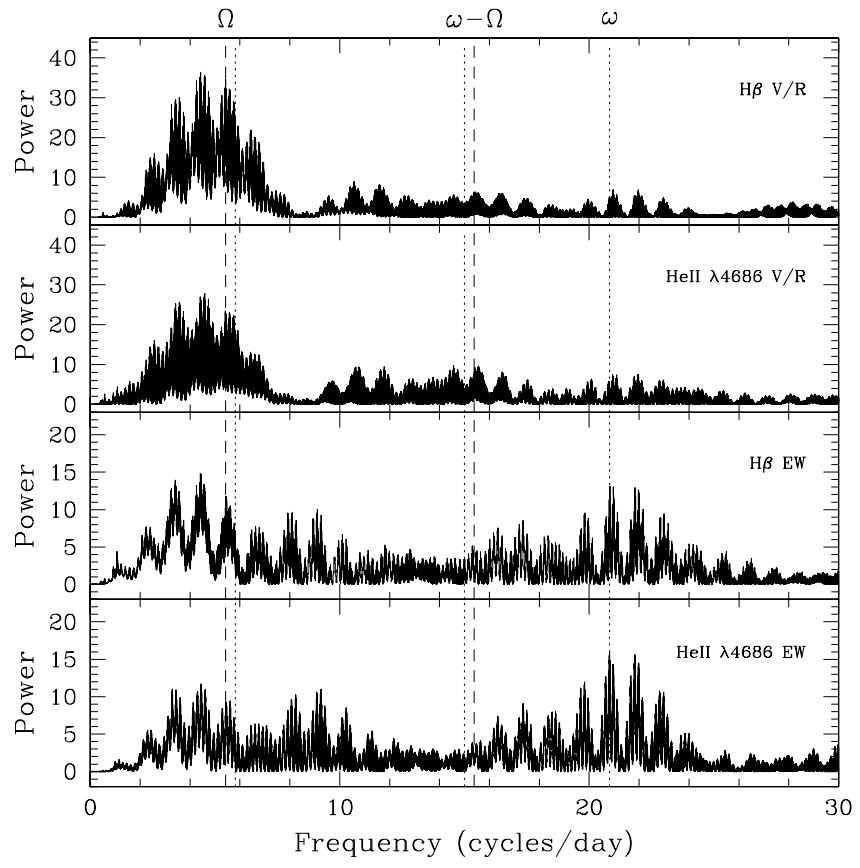

Fig. 11. Scargle periodograms of the $\mathrm{H} \beta$ and He II $\lambda 4686 V / R$ and $E W$ curves. Dashed (dotted) vertical lines correspond to spectroscopic (photometric) results.

\section{Discussion}

\subsection{The intermediate polar nature of HS $0943+1404$}

The combined photometric and spectroscopic results clearly qualify HS 0943+1404 as an asynchronous magnetic CV, an IP. The system has a likely orbital period of $P_{\text {orb }} \simeq 250 \mathrm{~min}$ and a white dwarf spin period of $P_{\text {spin }}=69.171 \pm 0.001 \mathrm{~s}$. The radial velocity analysis of our spectroscopy suggests $P_{\text {orb }}=265.2 \mathrm{~min}$, while the more extensive photometry suggests $P_{\text {orb }}=247.5 \mathrm{~min}$ from the detection of the beat and spin periods.

The combination of orbital and spin periods found in HS 0943+1404 is rather unusual, as it yields a large $P_{\text {spin }} / P_{\text {orb }} \simeq 0.3$ and a long orbital period. The only other known IPs with even larger $P_{\text {spin }} / P_{\text {orb }}$ are EXHya (Vogt et al. 1980), V1025 Cen (Buckley et al. 1998), and DW Cnc (Rodríguez-Gil et al. 2004a), all three are short-period systems below the period gap. The majority of IPs are concentrated near $P_{\text {spin }} / P_{\text {orb }} \simeq 0.1$ (see e.g. Barrett et al. 1988; King \& Lasota 1991; Warner \& Wickramasinghe 1991), but King \& Wynn (1999) showed that spin-orbit equilibria exist with $P_{\text {spin }} / P_{\text {orb }}>0.1$, explaining cases such as EX Hya. Most recently, Norton et al. (2004) demonstrated that a large range of permitted spin-orbit equilibria exists in the $\left(P_{\text {spin }} / P_{\text {orb }}, \mu_{1}\right)$ parameter space, with $\mu_{1}$ the magnetic moment of the white dwarf primary star. We used their Fig. 2 to estimate a magnetic moment of $\mu_{1} \sim 10^{34} \mathrm{G} \mathrm{cm}^{3}$ for HS $0943+1404$. This value is in the typical range found for polars, and assuming an average white dwarf $\left(M_{\mathrm{wd}} \simeq 0.65 M_{\odot}, R_{\mathrm{wd}} \simeq 10^{8} \mathrm{~cm}\right)$ would result in a magnetic dipole field strength of $\sim 20 \mathrm{MG}$. Hence, it appears entirely possible that HS $0943+1404$ is a true "intermediate" polar, that will synchronise once its orbital period and mass transfer rate decrease sufficiently - in fact, for the parameters determined here, HS 0943+1404 lies above the synchronisation line in Fig. 4 of Norton et al. (2004).

Another property that HS $0943+1404$ shares with polars is the occurence of deep ( $\simeq 3 \mathrm{mag}$ ) low states, such as the one observed in January 2003 (Table 1). Such low states are characteristic of polars, only one long-period IP is known to have entered a deep low state (see e.g. Warner 1999).

\subsection{Spin-beat period switching}

Our photometric data show that HS 0943+1404 switches between states which are either dominated by variability at the white dwarf spin period, or by variability at the beat period. Changes in the relative power of the spin signal with respect to the side-band signals have been observed in other IPs, such as TX Col (Buckley \& Tuohy 1989; Norton et al. 1997), and in V1062 Tau, where at a given time only the spin or the beat period is detected (Lipkin et al. 2004). In V1062 Tau, Lipkin et al. (2004) suggested that the switching between spin- and beat-dominated variability is triggered by changes in the system brightness which they relate to changes in the mass transfer rate.

A dominating signal at the beat period has been interpreted as a sign of disc-less accretion (e.g. Buckley et al. 1995; Hellier 2002, and references therein). Spin-dominated variability is generally explained by disc-fed accretion. The variations are thought to arise from reprocessing of X-rays at either the inner edge or the outer rim of the magnetically-truncated disc (Warner 1986), or from aspect variations of the accretion funnel feeding material from the inner disc edge onto the white dwarf (see e.g. Hellier et al. 1987).

The alternating dominance of the spin and beat signals in the light curves of HS 0943+1404 clearly implies changes in the accretion mode. However, in this system, both states have been observed at similar mean brightness levels (Table 1, Figs. 4 and 5). The fact that the spectrum of the secondary star is not detected even when the system is faint (although no spectrum in low state is available; see Fig. 7) indicates that the accretion disc must dominate the observed continuum. Hence, a predominant signal at the beat period cannot be unambiguously linked to disc-less accretion. All of this suggests that changes in the accretion mode are not solely due to variations in the mass transfer rate, but can also be triggered by other mechanisms within the binary system (Norton et al. 1997).

\subsection{An elevated nitrogen abundance}

The optical spectrum of HS $0943+1404$ is similar to that of the IP 1RXS J062518.2+733433 (=HS 0618+7336; AraujoBetancor et al. 2003). Both systems show unusually strong emission of $\mathrm{N}_{\text {II }} \lambda 5680$ compared to most other CVs. We interpret this as evidence for an enhanced nitrogen abundance in these two IPs, which is usually associated with the existence of CNO-processed material in the envelope of the donor star. Two possible scenarios can explain the origin of the CNOprocessed material in the envelope of the donor: either the 
companion has accreted material from the shell associated with a nova explosion on the white dwarf, or the secondary star is evolved (and the white dwarf is accreting material from the exposed CNO core whose outer layers were stripped off during a thermal time-scale mass-transfer phase, see Schenker et al. 2002; Podsiadlowski et al. 2003). Based on a HST FUV survey of cataclysmic variables, Gänsicke et al. (2003) have recently shown that $10 \%-15 \%$ of all CVs display enhanced nitrogen abundances. This is in agreement with the predictions by Schenker et al. (2002) and Podsiadlowski et al. (2003) that a non-negligible fraction of all CVs should have evolved through a phase of thermal time scale mass transfer.

\section{Conclusions}

The results presented in this paper are summarised as follows:

1. Based on our photometric and spectroscopic observations, we have shown that HS $0943+1404$ is a new intermediate polar CV with an orbital period of $P_{\text {orb }} \simeq 250 \mathrm{~min}$ and a white dwarf spin period of $P_{\text {spin }}=69.171 \pm 0.001 \mathrm{~s}$. An additional confirmation of the spin period, e.g. through the detection of a coherent variablity in X-rays or polarised light would be desirable.

2. Based on Norton et al.'s (2004) calculations, we estimate the magnetic moment of the white dwarf in HS 0943+1404 to be $\mu_{1} \sim 10^{34} \mathrm{G} \mathrm{cm}^{3}$, which is in the range of polars. Long-term photometric monitoring of the system revealed the occurrence of deep low states, during which the brightness drops by $\sim 3 \mathrm{mag}$, also typical of polars. It appears likely that HS $0943+1404$ is a true "intermediate" polar on its way to synchronization.

3. The optical spectrum of HS $0943+1404$ contains a number of unusual emission lines, most noticeably $\mathrm{N}_{\text {II }} \lambda 5680$, which suggests the presence of CNO-enriched material in the envelope of the secondary star. Ultraviolet spectroscopy of HS 0943+1404 would be useful to assess the carbon and nitrogen abundances from the $\mathrm{N} v \lambda 1240$ and $C_{\text {IV }} \lambda 1550$ resonance lines.

Acknowledgements. We wish to thank the anonymous referee for his/her contribution to the paper. We are grateful to Sergio Fernández for carrying out some of the IAC80 telescope observations. PRG and BTG thank PPARC for support through a PDRA and an AF, respectively. The HQS was supported by the Deutsche Forschungsgemeinschaft through grants Re 353/11 and Re 353/22. This work was supported in part by NASA grant NAG5-9930. The use of the MOLLY package developed and maintained by Tom Marsh is acknowledged.

\section{References}

Andronov, N., Pinsonneault, M., \& Sills, A. 2003, ApJ, 582, 358

Araujo-Betancor, S., Gänsicke, B. T., Hagen, H.-J., et al. 2005, A\&A, 430,629

Araujo-Betancor, S., Gänsicke, B. T., Hagen, H.-J., Rodríguez-Gil, P., \& Engels, D. 2003, A\&A, 406, 213

Barrett, P., O'Donoghue, D., \& Warner, B. 1988, MNRAS, 233, 759

Bertin, E., \& Arnouts, S. 1996, A\&AS, 117, 393
Buckley, D. A. H., Cropper, M., Ramsay, G., \& Wickramasinghe, D. T. 1998, MNRAS, 299, 83

Buckley, D. A. H., Sekiguchi, K., Motch, C., et al. 1995, MNRAS, 275, 1028

Buckley, D. A. H., \& Tuohy, I. R. 1989, ApJ, 344, 376

Gänsicke, B. T. 2004, in Compact Binaries in the Galaxy and Beyond, ed. G. Tovmassian, \& E. Sion, Conf. Ser. 20, Rev. Mex. Astron. Astrofís., 152

Gänsicke, B. T., Araujo-Betancor, S., Hagen, H.-J., et al. 2004, A\&A, 418,265

Gänsicke, B. T., Szkody, P., de Martino, D., et al. 2003, ApJ, 594, 443

Gänsicke, B. T., Fried, R. E., Hagen, H.-J., et al. 2000, A\&A, 356, L79

Gänsicke, B. T., Beuermann, K., \& Reinsch, K., ed. 2002a, The Physics of Cataclysmic Variables and Related Objects, ASP Conf. Ser., 261

Gänsicke, B. T., Hagen, H. J., \& Engels, D. 2002b, in The Physics of Cataclysmic Variables and Related Objects, ed. B. T. Gänsicke, K. Beuermann, \& K. Reinsch, ASP Conf. Ser., 261, 190

Hagen, H. J., Groote, D., Engels, D., \& Reimers, D. 1995, A\&AS, 111,195

Hellier, C. 2002, in The Physics of Cataclysmic Variables and Related Objects, ed. B. T. Gänsicke, K. Beuermann, \& K. Reinsch, ASP Conf. Ser., 261, 92

Hellier, C., Mason, K. O., Rosen, S. R., \& Cordova, F. A. 1987, MNRAS, 228, 463

Horne, K. 1986, PASP, 98, 609

Howell, S. B., Rappaport, S., \& Politano, M. 1997, MNRAS, 287, 929

Jiang, X. J., Engels, D., Wei, J. Y., Tesch, F., \& Hu, J. Y. 2000, A\&A, 362,263

King, A. R. 1988, QJRAS, 29, 1

King, A. R., \& Lasota, J. P. 1991, ApJ, 378, 674

King, A. R., \& Schenker, K. 2002, in The Physics of Cataclysmic Variables and Related Objects, ed. B. T. Gänsicke, K. Beuermann, \& K. Reinsch, ASP Conf. Ser., 261, 233

King, A. R., \& Wynn, G. A. 1999, MNRAS, 310, 203

Kolb, U. 1993, A\&A, 271, 149

Kolb, U., \& Baraffe, I. 1999, MNRAS, 309, 1034

Lipkin, Y. M., Leibowitz, E. M., \& Orio, M. 2004, MNRAS, 349, 1323

Norton, A. J., Hellier, C., Beardmore, A. P., et al. 1997, MNRAS, 289 , 362

Norton, A. J., Wynn, G. A., \& Somerscales, R. V. 2004, ApJ, 614, 349

Podsiadlowski, P., Han, Z., \& Rappaport, S. 2003, MNRAS, 340, 1214

Reimers, D., \& Hagen, H. J. 2000, A\&A, 358, L45

Reimers, D., Hagen, H. J., \& Hopp, U. 1999, A\&A, 343, 157

Rodríguez-Gil, P., Gänsicke, B. T., Araujo-Betancor, S., \& Casares, J. 2004a, MNRAS, 349, 367

Rodríguez-Gil, P., Gänsicke, B. T., Barwig, H., Hagen, H.-J., \& Engels, D. 2004b, A\&A, 424, 647

Scargle, J. D. 1982, ApJ, 263, 835

Schenker, K., \& King, A. R. 2002, in The Physics of Cataclysmic Variables and Related Objects, ed. B. T. Gänsicke, K. Beuermann, \& K. Reinsch, ASP Conf. Ser., 261, 242

Schenker, K., King, A. R., Kolb, U., Wynn, G. A., \& Zhang, Z. 2002, MNRAS, 337, 1105

Schneider, D. P., \& Young, P. 1980, ApJ, 238, 946

Schwarzenberg-Czerny, A. 1989, MNRAS, 241, 153

Thorstensen, J. R., \& Fenton, W. H. 2002, PASP, 114, 74

Vogt, N., Krzeminski, W., \& Sterken, C. 1980, A\&A, 85, 106

Warner, B. 1986, MNRAS, 219, 347

Warner, B. 1999, in Annapolis Workshop on Magnetic Cataclysmic Variables, ed. C. Hellier, \& K. Mukai, ASP Conf. Ser., 157, 63

Warner, B., \& Wickramasinghe, D. T. 1991, MNRAS, 248, 370 